\title{
Who needs a permanent pacemaker after transcatheter aortic valve implantation?
}

\author{
Bacik $\mathrm{P}^{1}$, Poliacikova $\mathrm{P}^{2}$, Kaliska $\mathrm{G}^{1}$ \\ Department of Arrhythmology, The Middle-Slovak Institute of Cardiovascular Diseases, \\ Banska Bystrica, Slovakia. bacik.peter@suscch.eu
}

\begin{abstract}
INTRODUCTION: We aimed to identify predictive factors for pacemaker requirement and determine the rate of pacemaker dependency in a single centre patient group.

METHODS AND REULTS: Out of 116 patients, who underwent transcatheter aortic valve implantation at our institution. Absence of atrial fibrillation at time of procedure, pulmonary hypertension, obesity and severity of aortic valve stenosis were predictive for the need of permanent pacemaker implantation after transcatheter aortic valve implantation.

CONCLUSION: Only persisting periprocedural and early-onset complete heart block were permanent and resulted in patients' pacemaker dependency. Definite predictors of persistence of conduction impairment are yet to be identified. We can predict only pacemaker dependency, but the real need of pacing in our risky patients with softer indications for permanent pacemaker after transcatheter aortic valve implantation cannot be clearly identified, essential in context of improving quality of their lives in avoiding symptomatic bradycardia, which can be intermittent, especially in such a group of elderly patients often with fragile and degenerate conduction system (Tab. 5, Ref. 34). Text in PDF www.elis.sk.

KEY WORDS: aortic stenosis, permanent pacemaker, transcatheter aortic valve implantation.
\end{abstract}

\section{Introduction}

Transcatheter aortic valve implantation (TAVI) has matured into the preferred treatment modality for patients with severe aortic stenosis at extreme or high risk for conventional surgery and a valuable alternative for those at intermediate risk in view of similar or superior clinical outcomes and decreased rates of periprocedural adverse events (1).

However, despite rapidly evolving implantation techniques, damage of the conduction system remains one of the major complications after TAVI. Depending on the type of valve prosthesis, up $20 \%$ of the patients undergoing TAVI require a permanent pacemaker (PPM (2)).

In previous studies, several patient and procedure-related factors have been shown to be associated with the need of PPM implantation after TAVI, including advanced age, male gender, atrial fibrillation, calcification of aortic and mitral annulus, small left ventricular outflow tract, preprocedural or intraprocedural conduction disorders, balloon pre-dilation, and depth of prosthesis implantation (3).

\footnotetext{
${ }^{1}$ Department of Arrhythmology, The Middle-Slovak Institute of Cardiovascular Diseases, Banska Bystrica, Slovakia, and ${ }^{2}$ Acute Cardiology Department, The Middle-Slovak Institute of Cardiovascular Diseases, Banska Bystrica, Slovakia

Address for correspondence: P. Bacik, MD, Department of Arrhythmology, The Middle-Slovak Institute of Cardiovascular Diseases, Banska Bystrica, Slovakia.
}

Because of its proximity to the aortic root, iatrogenic injury to the atrioventricular conduction system occurs also after surgical bioprosthesis implantation, however, according to the current guidelines, PPM implantation after SAVR should be deferred to 7 days after the procedure, since AV block after surgery may be attributed to electrolyte abnormalities or tissue oedema and therefore temporary (4). In the setting of TAVI, conduction disturbances result primarily from a direct mechanical insult to the conduction system associated with various degrees of oedema, hematoma, and ischemia (5). Current data suggest that conduction impairment may be reversible and timing of PPM implantation after TAVI remains a subject of debates.

In this study we aimed to identify predictive factors for pacemaker requirement as well as the rate of pacemaker dependency after TAVI in our centre patient population.

\section{Methods}

\section{Study population}

Between August 2013 and March 2017, we performed TAVI in 116 symptomatic patients with severe aortic stenosis. A decision to perform TAVI was made by heart team consisting of interventional cardiologist, cardiac surgeon and imaging specialist. Transfemoral TAVI approach was used in 96 patients $(82.8 \%)$, the remaining 20 patients were treated transapically (17.2\%). Sapien XT was implanted in 11 patients $(9.5 \%)$ and in majority of patients, Sapien 3 was used $(n=105,90.5 \%)$. 


\section{Pacemaker implantation}

We implanted 12 single chamber pacemakers, 3 dual chamber pacemakers and 1 biventricular pacemaker. Indications for pacemaker implantations were: complete AV block (intermittent or persistent) in 7 pts, symptomatic AV block gr. II in 1 of the patients, LBBB and substantial PQ interval prolongation in 4 pts, trifascicular block in 1 and alternans block in another patient. Biventricular pacemaker was used in 1 patient with new onset LBBB and reduction of left ventricular ejection fraction.

Timing of PPM implantation was 5.5 days $(\mathrm{SD} \pm 2.2)$.

The follow-up was scheduled to 3, 6 and 12 months after TAVI.

\section{Statistical analysis}

We used a logistic regression analysis to identify the predictors for pacemaker implantation.

\section{Results}

Our study population consisted of 69 females $(59.5 \%)$ and 47 males $(40.5 \%)$. The preoperative patients' characteristics are listed in Table 1.

The mean age was $77.1 \pm 5.7$ years. EUROSCORE II values were $4.6 \% \pm 3$.

Pre-TAVI ECG features of patients subsequently requiring PPM are listed in Table 2. Half of them had normal ECG, 1 patient had prolongation of PQ interval, one had intraventricular conduc-

Tab. 1. Baseline clinical preoperative patients' characteristics.

\begin{tabular}{lc}
\hline Variable & Value \\
\hline Total study group & $116(100 \%)$ \\
\hline Age (years) & $77.1 \pm 5.7$ \\
\hline Sex, women/men & $69(59.5 \%) / 47(40.5 \%)$ \\
\hline Weight $(\mathrm{kg})$ & $79.4 \pm 16.8$ \\
\hline Height $(\mathrm{cm})$ & $163.5 \pm 8.2$ \\
\hline EuroScore II & $4.6 \% \pm 3$ \\
\hline LVEF $(\%)$ & $50.5 \pm 9.0$ \\
\hline NYHA class I/I/III/IV & $1(0.9 \%) / 26(22.4 \%) / 70(60.3 \%) / 19(16.4 \%)$ \\
\hline AVA (cm2) & $0.7 \pm 0.2$ \\
\hline Aorta calcification & $5(4.3 \%)$ \\
\hline CAD, 1-VD/2-VD/3-VD & $22(19 \%) / 18(15.5 \%) / 18(15.5 \%)$ \\
\hline Prior CVA, TIA/with full & $7(6 \%) / 16(13.8 \%) / 4(3.4 \%)$ \\
recovery/with residual deficit & $23(19.8 \%) / 42(36.2 \%) / 18(15.5 \%)$ \\
\hline Pulmonary hypertension & $50(43.1 \%) / 60(51.7 \%) / 5(4.3 \%)$ \\
mild/moderate/severe & $37(31.9 \%) / 79(68.1 \%)$ \\
\hline Mitral regurgitation & $47(40.5 \%)$ \\
\hline mild/moderate/severe & $66(56.9 \%)$ \\
\hline Rhythm before procedure, AF/SR & $25(21.6 \%)$ \\
\hline Diabetes mellitus & $6(5.2 \%)$ \\
\hline Renal insufficiency & $8(6.9 \%)$ \\
\hline Pulmonary disease & Hepatal disease \\
\hline Poor mobility &
\end{tabular}

$\overline{\mathrm{AF}}=$ atrial fibrillation, $\mathrm{CAD}=$ coronary artery disease, $\mathrm{CVA}=$ cerebrovascular accident, $\mathrm{LVEF}=$ left ventricular ejection fraction, $\mathrm{NYHA}=$ New York Heart Association, $\mathrm{SR}=$ sinus rhythm, TIA = transitory ischaemic attack, $\mathrm{VD}=$ vessel disease
Tab. 2. ECG features before TAVI in patients with PPM after TAVI.

\begin{tabular}{lc}
\hline ECG & Number of patients - total $16(100 \%)$ \\
\hline Normal & $8(50 \%)$ \\
PQ interval prolongation & $1(6.3 \%)$ \\
LAH & $3(18.7 \%)$ \\
LBBB & $3(18.7 \%)$ \\
IVCD & $1(6.3 \%)$ \\
\hline
\end{tabular}

$\mathrm{ECG}=$ electrocardiogram, $\mathrm{IVCD}=$ intraventricular conduction disorder, $\mathrm{LAH}=$ left anterior hemiblock, $\mathrm{LBBB}=$ left bundle branch block, $\mathrm{PPM}=$ permanent pacemaker, TAVI $=$ transcatheter aortic valve implantation

Tab. 3. Preoperative clinical patients' characteristics.

\begin{tabular}{lc}
\hline Variable & Value \\
\hline Total study group & $116(100 \%)$ \\
Age (years) & $77.1 \pm 5.7$ \\
Sex, women/men & $69(59.5 \%) / 47(40.5 \%)$ \\
Weight $(\mathrm{kg})$ & $79.4 \pm 16.8$ \\
Height $(\mathrm{cm})$ & $163.5 \pm 8.2$ \\
EuroScore II & $4.6 \% \pm 3$ \\
LVEF $(\%)$ & $50.5 \pm 9.0$ \\
NYHA class I/II/III/IV & $1(0.9 \%) / 26(22.4 \%) / 70(60.3 \%) / 19(16.4 \%)$ \\
AVA $(\mathrm{cm} 2)$ & $0.7 \pm 0.2$ \\
Aorta calcification & $5(4.3 \%)$ \\
\hline
\end{tabular}

$\mathrm{AVA}=$ aortic valve area, $\mathrm{LVEF}=$ left ventricular ejection fraction, $\mathrm{NYHA}=\mathrm{New}$ York Heart Association

Tab. 4. Indications for pacemaker after TAVI.

\begin{tabular}{lc}
\hline Indication & Number of patients - total 16(100\%) \\
\hline AVB gr. II & $1(6.3 \%)$ \\
AVB gr. III & $7(43.5 \%)$ \\
LBBB with PQi prolongation & $4(25 \%)$ \\
SSS brady-tachy & $1(6.3 \%)$ \\
RBBB + LAH + PQi prolongation & $1(6.3 \%)$ \\
Alternans block (LBBB with RBBB) & $1(6.3 \%)$ \\
LBBB with reduction of LVEF & $1(6.3 \%)$ \\
\hline
\end{tabular}

$\overline{\mathrm{AVB}}=$ atrioventricular block, $\mathrm{LAH}=$ left anterior hemiblock, $\mathrm{LBBB}=$ left bundle branch block, $\mathrm{PPM}=$ permanent pacemaker, $\mathrm{LVEF}=$ left ventricle ejection fraction, $\mathrm{RBBB}=$ right bundle branch block, SSS $=$ sick sinus syndrome, TAVI $=$ transcatheter aortic valve implantation

tion disorder, 3 pts left anterior hemiblock and 3 pts left bundle branch block (Tab. 3).

In Table 4, we can see indications for implantation of PPM after TAVI. The major indication was a complete AV block (intermittent or persistent) in 7 pts, we implanted PPM in 1 patient with symptomatic AV block gr. II, in 4 pts with LBBB and substantial PQ interval prolongation, in 1 patient with trifascicular block and in 1 patient with alternans block. Biventricular pacemaker was used in 1 patient with new onset of LBBB and reduction of left ventricular ejection fraction.

We used a logistic regression analysis to identify, which factors are the most predictive for pacemaker implantation (Tab. 5). The strongest predictors were weight $(\mathrm{p}=0.086)$, absence of atrial fibrillation $(\mathrm{p}=0.028)$, aortic peak gradient $(\mathrm{p}=0.073)$, aortic valve area $(p=0.088$ and the severity of pulmonary hypertension $(\mathrm{p}=0.066)$.

The follow-up was performed at 3,6 and 12 months after TAVI. Pacemaker dependence (defined as more than $95 \%$ pacing events) was observed only in 3 pts (18.8 \% of PPM pts, $2.6 \%$ of 
Tab. 5. Significance of factors for pacemaker implantation after TAVI.

\begin{tabular}{|c|c|c|c|c|c|}
\hline & B & S.E. & Wald & $\mathrm{df}$ & Sig. \\
\hline age & .056 & .104 & .291 & 1 & .589 \\
\hline gender & -.401 & 1.434 & .078 & 1 & .780 \\
\hline height & -.117 & .077 & 2.339 & 1 & .126 \\
\hline weight & .079 & .046 & 2.949 & 1 & .086 \\
\hline euroskore II & -.024 & .219 & .012 & 1 & .914 \\
\hline diabetes & -.463 & 1.015 & .208 & 1 & .649 \\
\hline CHRI & .929 & 1.273 & .532 & 1 & .466 \\
\hline PD (pulmonary disease) & -1.176 & 1.612 & .532 & 1 & .466 \\
\hline HD (hepatal disease) & -17.363 & 14057.265 & .000 & 1 & .999 \\
\hline stroke & & & .356 & 3 & .949 \\
\hline PAO & .639 & 1.824 & .123 & 1 & .726 \\
\hline poor mobility & 2.253 & 1.848 & 1.486 & 1 & .223 \\
\hline calcification of aorta & -.262 & 3.221 & .007 & 1 & .935 \\
\hline rhythm before procedure & -3.634 & 1.650 & 4.853 & 1 & .028 \\
\hline NYHA & & & 3.312 & 3 & .346 \\
\hline CAD & & & 1.595 & 3 & .661 \\
\hline AoPG & .053 & .030 & 3.210 & 1 & .073 \\
\hline AVA & 7.405 & 4.344 & 2.906 & 1 & .088 \\
\hline MR & & & 2.735 & 3 & .434 \\
\hline $\mathrm{PH}$ & & & 7.205 & 3 & .066 \\
\hline LVEF & & & 1.084 & 2 & .581 \\
\hline approach & -.440 & 2.395 & .034 & 1 & .854 \\
\hline valve model & 2.162 & 2.218 & .950 & 1 & .330 \\
\hline PG after procedure & -.015 & .072 & .044 & 1 & .834 \\
\hline bleeding after procedure & & & 1.668 & 3 & .644 \\
\hline Constant & -28.408 & 56842.000 & .000 & 1 & 1.000 \\
\hline
\end{tabular}

$\mathrm{AVA}=$ aortic valve area, $\mathrm{AoPG}=$ aortic peak gradient, $\mathrm{CAD}=$ coronary artery disease, $C H R I=$ chronic renal insufficiency, $L V E F=$ left ventricular ejection fraction, $\mathrm{MR}=$ mitral regurgitation, $\mathrm{NYHA}=$ New York Heart Association, $\mathrm{PG}=$ peak gradient, $\mathrm{PH}=$ pulmonary hypertension, $\mathrm{TAVI}=$ transcatheter aortic valve implantation

total pts) during each follow up, 3, 6 and 12 months (two of them with postprocedural persistent AVB III and one with new onset LBBB and extreme long PQ interval more than $360 \mathrm{~ms}$ ). However, 1 of them was found to be falsely dependent on stimulation (>99\% pacing events), which was corrected by using AV search hysteresis to facilitate native AV conduction. At 12 months follow up, less than $5 \%$ pacing events occurred in 3 pts $(18.8 \%$ of PPM pts, $2.6 \%$ of total pts) with LBBB + 1st degree AVB at. 2 pts ( $12.5 \%$ of PPM pts, $1.7 \%$ of total pts) died (the causes of death are unknown) within one year after TAVI. So, the real dependency on PPM at 12 months follow-up was demonstrated just in 2 cases (12.5\% of PPM pts, $1.7 \%$ of total pts) with postprocedural persistent complete AV block.

\section{Discussion}

The majority of cases of PPM implantation post TAVI are due to a complete or high-degree atrioventricular (AV) block (> 80\%) (3). Certain types of valves (e.g. self-expandable CoreValve prosthesis) are associated with higher rates of PPM implantation (6). At our institution, we only used balloon expandable prostheses Sapien XT and Sapien 3, without statistical difference in terms of permanent pacemaker need between these two valves.

About half of incidences of PPM implantation in the PARTNER trial and registry were within 48 hours of the procedure, 86 $\%$ within a week, and almost all of them $(97.1 \%)$ in the index hospitalization, with only $1.9 \%$ of the patients receiving a PPM in
1 year (3). Urena et al reported the median time of PPM implantation of 3 days, with approximately $90 \%$ of implantations within a week. However, more than a third of patients, who received a PPM, did not show pacing activity at 6 - and 12-month follow-up and no association was observed between the need for 30-day PPM and all-cause mortality, cardiovascular mortality and allcause mortality or rehospitalization due to heart failure, although new PPM did have a negative effect on left ventricular function over time. On the other hand, a lower rate of sudden or unknown death has been reported in patients with PPM (7).

The long-term persistence of AVB III is generally low after TAVI. Gaede et al documented pre-existing right bundle branch block and implantation of a CoreValve prosthesis as the strongest independent predictors of PPM implantation after TAVI, whereby the leading indication for PPM implantation was also AVB III. But only $22.4 \%$ of these patients had a persistent AVB III at followup during 3 months. Predictors of AVB III persistence were prior RBBB, post-dilatation and higher mean aortic valve gradient prior to implantation. Early PPM implantation after TAVI (1 day after procedure) was the only independent predictor of persistent AVB III (2), like in our cohort. Another study by Naveh et al also showed that pre-existing RBBB, baseline PR interval, post-TAVR PR interval, and change in PR interval from baseline are reliable predictors of long-term PPM dependency (8). So, we have sufficient evidences of the role of a pre-existing RBBB in the development of cardiac conduction disorders after TAVI $(3,9,10)$. The mechanical stress of the implanted prosthesis mainly affects the left bundle branch, which explains that a pre-damaged right bundle branch predisposes to a complete AVB, when exposed to the shear stress of the TAVI prosthesis (11).

In contrast to these previously published data, in our patient cohort, pre-existing RBBB and atrial fibrillation were not determinant of pacemaker requirement (because of small number of patients and absence of these features in PPM patients before TAVI). The severity of aortic stenosis was predictive of pacemaker need, which is not surprising in the context of previously described negative impact of severe calcification in this regard.

In our single centre analysis, novel potential post-TAVI conduction impairment predictors (obesity and pulmonary hypertension) have been identified.

The classification of patients according to ventricular pacing (VP) $\geq 95 \%$ and VP $\leq 5 \%$ is the cut-off indicating an absolute dependency and absolute independency; nevertheless, patients with $\mathrm{VP} \leq 5 \%$ could still need a PPM to prevent syncope or cardiac arrest. Additionally, the programming in some patients presenting with AVB III in the first control after implantation might result in a false high ventricular pacing rate in some patients (2). Pacemaker dependence in our pts was observed only in 3 pts $(18.8 \%$ of PPM pts, $2.6 \%$ of total pts) during each follow up, 3,6 and 12 month (two of them with postprocedural persistent AVB III and one with new onset LBBB and extreme long PQ interval more than $360 \mathrm{~ms}$ ). However, 1 patient reported a false dependency on stimulation (>99\% pacing events), which was adjusted by using of AV search hysteresis to prefer native AV conduction. So, the real dependency on PPM at 3, 6 and 12 months follow-up was 
demonstrated just in 2 cases $(12.5 \%$ of PPM pts, $1,7 \%$ of total pts) with postprocedural persistent AVB III and very early time of PPM implantation (2 days after TAVI). On the other hand, less than $5 \%$ pacing events occurred in 3 pts $(18.8 \%$ of PPM pts, 2.6 $\%$ of total pts) with LBBB with PQi prolongation after all follow ups. However, it does not really mean that there was no need for insertion of PPM in the rest of pts, because majority of them reported an intermittent pacing (between $19 \%$ and $40 \%$ pacing events) and tailored programming for every single patient can improve their quality of life avoiding symptomatic bradycardia.

While post TAVI complete and high grade heart block have been generally accepted as definite indications for permanent pacing, what to do with less severe conduction impairment still remains a matter of debate. The management of LBBB occurring after TAVI remains controversial and differs from centre to centre because of the absence of strict recommendations by international guidelines. Several previous studies have shown that pre-existing and new BBBs (LBBBs and RBBBs) were significant predictive factors for PPM implantation, whereas others have shown the opposite result (12). The progression of LBBB to high-degree AVB should trigger the indication for PPM. However, LBBB will persist in most patients with no further changes (progression or regression) during the hospitalization period (13). It is known in general that the implantation of a pacemaker in patients with bundle branch block (no matter the TAVI) in the case of syncope associated with the $\mathrm{HV}$ interval $>70 \mathrm{~ms}$ and in the presence of the HV interval > $100 \mathrm{~ms}$ if the patient is asymptomatic is recommended (4). QRS prolongation after TAVI procedure, mostly related to new-onset LBBB, has been shown to be predictive of pacemaker implantation according to some smaller studies (14). Prolongation of intracardiac conduction times during EPS has been identified during and after TAVI implantation procedures (15). However, a strategy using EPS and remote monitoring of cardiovascular implantable electronic devices (CIEDs) in patients with an asymptomatic conduction disturbance (LBBB) after TAVI has yet to be validated. In their ongoing study, Massoullié et al evaluate patients with persisting LBBB after TAVI (16). The exact role of EPS and implantable loop recorders in these cases remains unclear. The other ongoing studies are the MARE study (Ambulatory Electrocardiographic Monitoring for the Detection of High-Degree Atrio-Ventricular Block in Patients With New-Onset Persistent Left Bundle-Branch Block After Transcatheter Aortic Valve Implantation; NCT02153307) and the Assessment of the Prognosis of Persistent LBBB After TAVI by an Electrophysiological and Remote Monitoring Risk Adapted Algorithm study (NCT02482844), among other awaited studies, will likely shed more light on the natural history of conduction disturbances after TAVI.

In our study, in 32 pts from all 116 pts appeared LBBB after TAVI (27.6\%), but only 5 pts of them received PPM at all (4.3\%). It is interesting that from 4 pts with LBBB and substantial PQ interval prolongation, 3 patients were not dependent from stimulation. This can cast doubt on relative indications for PPM in the context of reversibility of these conduction disorders. But we also know that LBBB can have a negative prognostic value documented in some previous works and these kinds of conduction disturbances more likely persist in older patients. As mentioned above, considering the higher risk of high-degree AVB within the months after the procedure in patients with new-onset persistent LBBB (17), some remote monitoring (implantable loop recorder or 24- to 48hour electrocardiographic monitoring at 30-day follow-up) may be advisable. This would also apply to those patients with LBBB and first-degree AVB, for whom an electrophysiological study or even PPM may be an option (18). However, the level of evidence for any of these measures remains low. Particular attention should be paid to the patients with new-onset LBBB and a prolonged QRS duration (particularly $>160$ milliseconds), which has been associated with an increased risk of overall mortality and sudden cardiac death (19). This subgroup may have a reasonable indication for PPM. Other prophylactic indications for PPM in the context of new-onset LBBB lack evidence and may lead to an excessive and inappropriate number of PPM. The potential complications associated with PPM should also be taken into account. It is associated with an increased cost, longer duration of hospitalization, and higher rates of repeat hospitalization and mortality or repeat hospitalization at 1 year (3). However, further studies are warranted.

So, in the view of lack of guidance, it has been left to the local protocols and individual physicians' decisions. No study to date has allowed definition of the predictors of the occurrence of a complete AV block or sudden cardiac death in a population of patients with LBBB after TAVI, although the potential value of EPS or implantable loop monitoring is currently being evaluated in clinical trials and their results are highly anticipated.

In our centre, new onset LBBB with wide QRS, progressively prolonging PR interval, especially in combination with pre-existing conduction disorders and/or transient periprocedural complete AV block, was one of the indications for pacing. Our findings in this study do not support such strategy though, 3 out of $4 \mathrm{pts}$ with this indication were found to be pacemaker independent during 12 months follow-up. In the light of these facts it may be wise to postpone the indication for PPM implantation for a couple of days.

Also, the correlation of QRS width with a complete block has also not been fully clarified (20). There are some clinical risk factors of new-onset LBBB after TAVI, which include the presence of preprocedural conduction abnormalities, especially prolonged QRS duration, female sex, previous coronary artery bypass graft (21), diabetes mellitus (22) and the amount of calcification of the aortic valve (23). In the largest meta-analysis to date, Regueiro et al (24) failed to show a significant association between the occurrence of TAVI-induced LBBB and 1-year all-cause mortality. In contrast, LBBB was associated with a higher risk of 1-year cardiac mortality. This relationship may be explained by a specific association between new-onset LBBB, especially when the QRS duration is $>160$ milliseconds, and the risk of sudden cardiac death during follow-up. Engborga et al observed the effect of PPM on mortality after TAVI. Almost third of the patients received a PPM within 30 days of the TAVI procedure. Kaplan-Meier survival analysis showed a higher mortality in patients without PPM. So TAVI-patients with a PPM had better survival than patients in whom a PPM was not implanted (25). 


\section{$560-565$}

Recent studies have also confirmed the strong association between frailty state and the 12-month mortality $(26,27)$. Frailty is a geriatric syndrome, which is characterized by a vulnerable health status associated with a declining function of multiple physiological systems and loss of physiological reserves with consecutive impairment of many domains (physical, social, nutritional, neuropsychological) (28). The presence of frailty has been associated with poor medical outcomes in different cardiac patients such as: patients with coronary artery disease (29) and chronic heart failure (30) as well as in patients undergoing TAVI. However, as this is multifactorial, frailty is difficult to capture, and it is timeconsuming to assess all components; it might be difficult to implement tests to determine this syndrome in clinical practice, such as at our institution.

In our cohort, 8 pts $(6.9 \%)$ died within the first 30 days after TAVI, one of them $(0.9 \%)$ received PPM (indication was AVB II and we documented PPM non-dependency). One year mortality data are not completely available at this time.

Schwerg et al took interest in the question: „When Is Pacemaker Implantation in pts with complete atrioventricular block after TAVI safe?"As we know, TAVI is routinely performed under anticoagulation using heparin, which potentially may lead to an increased bleeding rate in patients, who undergo PPM implantation immediately after TAVI. They retrospectively collected data on PPM implantations after TAVI during 3 years and proved that PPM implantation even next day after TAVI is safe (31).

Timing of PPM implantation in our study was 5.5 days after TAVI $(\mathrm{SD} \pm 2.2)$ with maximum 9 days and minimum 2 days. As mentioned above, it may be wise to postpone the indication for PPM implantation for a couple of days in some cases. On the other hand, nowadays is a tendency to shorten the time of hospitalization, which might be a limitation of this "waiting strategy".

\section{Conclusion}

TAVI is an expanding catheter-based technology that allows for implantation of a prosthetic valve without requiring openheart surgery for treatment of severe aortic stenosis. Large registries and randomized trials (32) have proved the effectiveness of this procedure, and the 1-year results appear favourable compared to surgery. Previously, TAVI was confined to the treatment of elderly and high-risk patients, however more recently, TAVI is increasingly used to treat the intermediate-risk patients. Improvement in valve implantation techniques, the wide dissemination of the procedure, operator experience and the development of new prostheses have dramatically reduced the rate of severe complications. One of the major complications of TAVI is the damage of the conduction system (33). The incidence of a de novo LBBB remains very high, ranging from $5 \%$ to $40 \%$ depending on the type of valve used (34). The 2013 European Society of Cardiology guidelines recommend a period of clinical observation up to 7 days for recovery before proceeding with PPM implantation in patients with a persistent high degree or complete AV block postsurgery or post-TAVI in order to assess whether the conduction disturbance is transient or permanent (Class I recommendation;
Level of Evidence C) (4). In all our cohort, LBBB occurred in $27.6 \%$ pts, but only $4.3 \%$ of them received PPM. The patients with soft indications for pacing (LBBB with PQ prolongation) were found to be pacemaker independent at 1 year follow-up. We showed, that the strongest predictors for PPM need after TAVI at our institution were the absence of atrial fibrillation at the time of TAVI, weight (the more obese patient, the higher the probability for a persistent bradycardia), aortic peak gradient before procedure (higher gradient means higher risk for pacemaker implantation), aortic valve area before procedure (smaller area is riskier) and the severity of pulmonary hypertension. We proved that TAVI is a safe and efficient method of treating severe aortic valve stenosis in selected patient population. Only persisting periprocedural and early-onset complete heart block were permanent and resulted in patients ' pacemaker dependency. For the time being, close monitoring of patients with known risk factors of postprocedural pacemaker requirement is warranted.

\section{References}

1. Siontis GC, Praz F, Pilgrim T et al. Transcatheter aortic valve implantation vs. surgical aortic valve replacement for treatment of severe aortic stenosis: a meta-analysis of randomized trials. Eur Heart J 2016; 37: 3503-3512.

2. Gaede L, Kim WK, Liebetrau Ch et al. Pacemaker implantation after TAVI: predictors of AV block persistence. Clin Res Cardiol 2018; 107 (1): 60-69.

3. Nazif TM, Dizon JM, Hahn RT et al. PARTNER Publications Office. Predictors and clinical outcomes of permanent pacemaker implantation after transcatheter aortic valve replacement: the PARTNER (Placement of AoRtic TraNscathetER Valves) trial and registry. JACC Cardiovasc Interv 2015; 8 (1 pt A): 60-69.

4. Brignole M, AuricchioA, Baron-Esquivias G et al. ESC guidelines on cardiac pacing and cardiac resynchronization therapy: the task force on cardiac pacing and resynchronization therapy of the European Society of Cardiology (ESC). Developed in collaboration with the European Heart Rhythm Association (EHRA). Europace 2013; 15: 1070-1018.

5. Moreno R, Dobarro D, López de Sá E et al. Cause of complete atrioventricular block after percutaneous aortic valve implantation: insights from a necropsy study. Circulation 2009; 120: e29-e30.

6. Mohr FW, Holzhey D, Möllmann H et al. The German Aortic Valve Registry: 1-year results from 13,680 patients with aortic valve disease. Eur J Cardio-thoracic Surg 2014; 46: 808-816.

7. Urena M, Webb JG, Tamburino C et al. Permanent Pacemaker Implantation Following Transcatheter Aortic Valve Implantation: Impact on Late Clinical Outcomes and Left Ventricular Function. Circulation 2014; 129: 1233-1243.

8. Naveh S, Perlman GY, Elitsur Y et al. Electrocardiographic Predictors of Long-Term Cardiac Pacing Dependency Following Transcatheter Aortic Valve Implantation. J Cardiovasc Electrophysiol 2017; 28: 216-223.

9. Erkapic D, De Rosa S, Kelava A et al. Risk for permanent pacemaker after transcatheter aortic valve implantation: a comprehensive analysis of the literature. J Cardiovasc Electrophysiol 2012; 23: 391-397.

10. Fraccaro C, Buja G, Tarantini G et al. Incidence, predictors, and outcome of conduction disorders after transcatheter self-expandable aortic valve implantation. Am J Cardiol 2011; 107: 747-754. 
11. Boerlage -VAN, Dijk K, Kooiman KM, Yong ZY et al. Predictors and permanency of cardiac conduction disorders and necessity of pacing after transcatheter aortic valve implantation. Pacing Clin Electrophysiol 2014; 37: 1520-1529.

12. Franzoni I, Latib A, Maisano F et al. Comparison of incidence and predictors of left bundle branch block after transcatheter aortic valve implantation using the CoreValve versus the Edwards valve. Am J Cardiol 2013; 112: 554-559.

13. Nazif TM, Williams MR, Hahn RT et al. Clinical implications of newonset left bundle branch block after transcatheter aortic valve replacement: analysis of the PARTNER experience. Eur Heart J 2014; 35: 1599-1607.

14. Khawaja MZ, Rajani R, Cook A et al. Permanent pacemaker insertion after CoreValve transcatheter aortic valve implantation: incidence and contributing factors (the UK CoreValve Collaborative). Circulation 2011; 123: 951-960.

15. Akin I, Kische S, Paranskaya $L$ et al. Predictive factors for pacemaker requirement after transcatheter aortic valve implantation. BMC Cardiovasc Disord 2012; 12: 87.

16. Massoullié G, Bordachar P, Irles D et al. Prognosis assessment of persistent left bundle branch block after TAVI by an electrophysiological and remote monitoring riskadapted algorithm: rationale and design of the multicentre LBBB-TAVI Study. BMJ Open 2016; 6: e010485.

17. Ando T, Takagi H; ALICE (All-Literature Investigation of Cardiovascular Evidence) Group. The prognostic impact of new-onset persistent left bundle branch block following transcatheter aortic valve implantation: a metaanalysis. Clin Cardiol 2016; 39: 544-550.

18. Tovia-Brodie O, Ben-Haim Y, Joffe $\mathbf{E}$ et al. The value of electrophysiologic study in decision-making regarding the need for pacemaker implantation after TAVI. J Interv Card Electrophysiol 2017; 48: 121-130.

19. Houthuizen P, Van Garsse LA, Poels TT et al. Left bundle branch block induced by transcatheter aortic valve implantation increases risk of death. Circulation 2012; 126: 720-728.

20. Meguro K, Lellouche N, Yamamoto M et al. Prognostic value of QRS duration after transcatheter aortic valve implantation for aortic stenosis using the CoreValve. Am J Cardiol 2013; 111: 1778-1783.

21. Schymik G, Tzamalis P, Bramlage $P$ et al. Clinical impact of a new left bundle branch block following TAVI implantation: 1-year results of the TAVIK cohort. Clin Res Cardiol 2015; 104: 351-362.

22. Houthuizen P, Van Garsse LA, Poels TT et al. Left bundlebranch block induced by transcatheter aortic valve implantation increases risk of death. Circulation 2012; 126: 720-728.
23. Hein-Rothweiler R, Jochheim D, Rizas K, Egger A, Theiss H, Bauer A, Massberg S, Mehilli J. Aortic annulus to left coronary distance as a predictor for persistent left bundle branch block after TAVI. Catheter Cardiovasc Interv 2017; 89: E162-E168.

24. Regueiro A, Abdul-Jawad Altisent O, Del Trigo $M$ et al. Impact of new-onset left bundle branch block and periprocedural permanent pacemaker implantation on clinical outcomes in patients undergoing transcatheter aortic valve replacement: a systematic review and meta-analysis. Circ Cardiovasc Interv 2016; 9: e003635.

25. Engborga J, Riechel-Sarupa C, Gerkeb $O$ et al. Effect of permanent pacemaker on mortality after transcatheter aortic valve replacement. Scandinavian Cardiovascular Journal 2016; 10: 40-46.

26. Kleczynski P, Dziewierz A, Bagienski M, et al. Impact of frailty on mortality after transcatheter aortic valve implantation. Am Heart J 2017; 185: $52-58$.

27. Shimura T, Yamamoto M, Kano S et al. Impact of the clinical frailty scale on outcomes after transcatheter aortic valve replacement. Circulation 2017; 135 (21): 2013-2024.

28. Afilalo J, Alexander KP, Mack MJ, Maurer MS, Green P, Allen LA, Popma JJ, Ferrucci L, Forman DE. Frailty assessment in the cardiovascular care of older adults. J Am Coll Cardiol 2014; 63 (8): 747-762.

29. Nunez J, Ruiz V, Bonanad C, Minana G, Garcia-Blas S, ValeroE, Nunez E, Sanchis J. Percutaneous coronary intervention and recurrent hospitalizations in elderly patients with non ST-segment acute coronary syndrome: The role of frailty. Int J Cardiol 2017; 228: 456-458.

30. Denfeld QE, Winters-Stone K, Mudd JO, Hiatt SO, Chien CV, Lee CS. Frequency of and significance of physical frailty in patients with heart failure. Am J Cardiol 2017; 119 (8): 1243-1249.

31. Schwerg M, Baldenhofer G, Dreger $\mathbf{H}$ et al. Complete Atrioventricular Block after TAVI: When Is Pacemaker Implantation Safe? PACE 2013; 36: 898-903.

32. Pighi M, Serdoz R, Kilic ID et al. TAVI: New trials and registries offer further welcome evidence - U.S. CoreValve, CHOICE, and GARY. Glob Cardiol Sci Pract 2014; 2014: 78-87.

33. Houthuizen $P$, vanderBoon RM, Urena $M$ et al. Occurrence, fate and consequences of ventricular conduction abnormalities after transcatheter aortic valve implantation. EuroIntervention 2014; 9: 1142-1150.

34. Erkapic D, De Rosa S, Kelava A et al. Risk for permanent pacemaker after transcatheter aortic valve implantation: a comprehensive analysis of the literature. J Cardiovasc Electrophysiol 2012; 23: 391-397.

Received April 11, 2018. Accepted May 23, 2018. 